\title{
The rice diacylglycerol kinase family: functional analysis using transient RNA interference
}

\author{
Hongliang Ge, Chu Chen, Wen Jing, Qun Zhang, Hong Wang, Rong Wang and Wenhua Zhang*
}

State Key Laboratory of Crop Genetics and Germplasm Enhancement, College of Life Sciences, Nanjing Agricultural University, Nanjing, China

Edited by:

Kent D. Chapman, University of North

Texas, USA

\section{Reviewed by:}

Shan Lu, Nanjing University, China

Yueyun Hong, Huazhong Agricultural

University, China

*Correspondence:

Wenhua Zhang, State Key Laboratory

of Crop Genetics and Germplasm

Enhancement, College of Life

Sciences, Nanjing Agricultural

University, Nanjing 210095, China.

e-mail:whzhang@njau.edu.cn
Diacylglycerol kinase (DGK) is a pivotal enzyme that phosphorylates diacylglycerol (DAG) to form phosphatidic acid (PA). The production of PA from phospholipase D (PLD) and the coupled phospholipase C/DGK route is an important signaling process in animal and plant cells. In this study, we report a genomic analysis of eight putative rice DGKs encoded by a gene family (OsDGKs) grouped into three clusters. To further investigate the functions of the OsDGKs, a double-stranded RNA (dsRNA)-induced RNA silencing method was established. Introduction of in vitro-synthesized dsRNAs corresponding to a unique or conserved region of OsDGKs into rice protoplasts abolished or diminished the expression of individual or multiple OsDGK genes. Suppressing the expression of OsDGKs resulted in a distinct depletion of the transcripts of the defense gene OsNPR1 and the salt-responsive gene OsCIPK15. Our primary results suggest that OsDGKs are involved in the signaling of stress responses.

Keywords: diacylglycerol kinase, double-stranded RNA, rice, RNA interference

\section{INTRODUCTION}

Phospholipase C (PLC) catalyzes the hydrolysis of phosphatidylinositol 4,5-bisphosphate [PtdIns(4,5)P2] to produce diacylglycerol (DAG), which is phosphorylated to phosphatidic acid (PA) by diacylglycerol kinases (DGKs; Arisz et al., 2009). In animal cells, DAG and PA are important signaling molecules. DGK is thought to act as a conversional switch with two functional implications: the termination of DAG signaling and the initiation of PA signaling (Frere and Paolo, 2009). However, no direct DAG target (such as PKC in animal cells) has been found in plants, and the role for DAG as a plant signaling molecule has yet to been confirmed (Munnik and Testerink, 2009). In contrast, PA generated from both the PLD and PLC/DGK pathways is emerging as a stress signal molecule in plants (Munnik and Testerink, 2009; Hong et al., 2010; Zhang et al., 2010). PLD-derived PA has been found to regulate a series of developmental and environmental responses via its downstream targets (Li et al., 2009; Zhang et al., 2010). Relatively little genetic evidence for PA from PLC-coupled DGK regulating signaling in plant cells has been found (Munnik and Testerink, 2009).

Diacylglycerol kinase activity has been reported in several plant species, including tobacco, wheat, tomato, and Arabidopsis (Kamada and Muto, 1991; Lundberg and Sommarin, 1992; Wissing and Wagner, 1992; Katagiri et al., 1996; Snedden and Blumwald, 2000). Recently, multiple DGK-encoding genes have been isolated from plants (Snedden and Blumwald, 2000; GómezMerino et al., 2005; Chen et al., 2007). In Arabidopsis, AtDGK2 and AtDGK7 have been biochemically characterized (Gómez-Merino et al., 2004, 2005). A hydrophobic segment at the $\mathrm{N}$-termini of AtDGK1 and AtDGK2 is necessary to target the resulting proteins to endoplasmic reticulum (ER) membranes (Vaultier et al., 2008).

In rice, pharmacological evidence indicates that PLC/DGKmediated signaling is required for a benzothiadiazole-induced oxidative burst and hypersensitive cell death, and the transcription of one OsDGK is induced during this process (Chen et al., 2007). No genomic analysis of the rice DGK family has been reported. In this study, we report that OsDGKs are grouped into three clusters (I, II, and III) based on gene architecture, evolutionary relationships, and sequence identity. The transcription of OsDGKs was characterized using RT-PCR and real-time PCR, and their expression levels following treatment with xylanase or salt were analyzed. We established a transient double-stranded RNA (dsRNA)-induced RNA silencing assay that was used for rapid analysis of OsDGK functions in stress responses.

\section{MATERIALS AND METHODS SUSPENSION CELL CULTURES AND TREATMENTS}

Rice (Oryza sativa L. Nipponbare ssp. japonica) suspension cells were initiated from embryogenic calli induced from mature rice scutella. The cells were grown in 100-ml conical flasks containing Murashige and Skoog liquid medium supplemented with $3 \%$ sucrose and $2 \mathrm{mg} / \mathrm{l} 2,4-\mathrm{D}$, and incubated on a rotary shaker (100-120 rpm) at $26 \pm 2^{\circ} \mathrm{C}$ in darkness. The suspension cells were subcultured every 7 day.

Fresh suspension cells were collected by centrifugation after subculture for 4 to 5 day, and then treated with $200 \mu \mathrm{g} / \mathrm{ml}$ xylanase (Trichoderma viride; Fluka Bio-Chemika).

\section{RICE SEEDLING GROWTH AND PROTOPLAST ISOLATION}

Rice seedlings were grown in a growth room at $27^{\circ} \mathrm{C}$ for $2-3$ weeks. Seedlings of $5-8$ " high were used for protoplast isolation according to the method of Sheen (2001) with modifications. Stems, including the sheaths of young seedlings, were cut into $\sim 0.5-$ $1.0 \mathrm{~mm}$ segments using a razor blade, and immediately placed in a Petri dish containing K3 medium (see Table A1 in Appendix) 
supplemented with $1.5 \%$ cellulase R-10 (Yakult Honsha, Japan) and $0.3 \%$ macerozyme R-10 (Yakult Honsha). The segments were vacuum-infiltrated for $1 \mathrm{~h}$ at $20 \mathrm{mmHg}$ and digested in darkness with gentle shaking $(\sim 40 \mathrm{rpm})$ at room temperature for about $4 \mathrm{~h}$. Following the incubation, the enzyme solution was gently removed using a glass pipette, and the same volume of W5 solution $(154 \mathrm{mM}$ $\mathrm{NaCl}, 125 \mathrm{mM} \mathrm{CaCl}_{2}, 5 \mathrm{mM} \mathrm{KCl}, 2 \mathrm{mM}$ MES, $5 \mathrm{mM}$ glucose, $\mathrm{pH}$ 5.6) was added to the Petri dish for further shaking ( $\sim 80 \mathrm{rpm})$ for $1 \mathrm{~h}$ to release the protoplasts. Both the enzyme solution and the W5 solution were filtered through a $35-$ to $75-\mu \mathrm{m}$ nylon mesh. The protoplasts were collected by centrifugation at $150 \times g$ for $4 \mathrm{~min}$ at room temperature. The pelleted protoplasts were washed twice with W5 solution and counted under a microscope using a hemocytometer.

\section{STRESS TREATMENTS}

Protoplasts $\left(1 \times 10^{5}\right)$ were incubated in a six-well dish with each well containing $2 \mathrm{ml}$ of WI culture medium ( $500 \mathrm{mM}$ mannitol, $4 \mathrm{mM}$ MES, $20 \mathrm{mM} \mathrm{KCl}, \mathrm{pH}$ 5.6) supplemented with $150 \mu \mathrm{g} / \mathrm{ml}$ xylanase. For salt treatment, protoplasts $\left(1 \times 10^{5}\right)$ were incubated in modified WI culture medium containing $50 \mathrm{mM} \mathrm{NaCl}(400 \mathrm{mM}$ mannitol, 4 mM MES, $20 \mathrm{mM} \mathrm{KCl,} 50 \mathrm{mM} \mathrm{NaCl}$, pH 5.6). WI culture medium was used as a control. The protoplasts were incubated at $28^{\circ} \mathrm{C}$ in darkness for the indicated times, followed by harvesting with centrifugation at $200 \times g$ for $5 \mathrm{~min}$.

\section{RNA ISOLATION, RT-PCR, AND REAL-TIME PCR}

Total RNAs were isolated from suspension cells or protoplasts using Trizol reagent according to the manufacturer's protocol (Takara, Japan). Reverse transcription was performed using Prime Script ${ }^{\text {TM }}$ RT Reagent Kit (Takara). RT-PCR conditions were as follows: denaturing at $95^{\circ} \mathrm{C}$ for $5 \mathrm{~min}$, followed by 30 cycles of $95^{\circ} \mathrm{C}$ for $30 \mathrm{~s}, 55^{\circ} \mathrm{C}$ for $30 \mathrm{~s}$, and $72^{\circ} \mathrm{C}$ for $30 \mathrm{~s}$, and a final extension at $72^{\circ} \mathrm{C}$ for $5 \mathrm{~min}$. The primers used for RT-PCR analyses are described in Table A2 in Appendix. The OsGAPDH gene was amplified as an internal control.

Real-time PCR conditions were as follows: denaturing at $95^{\circ} \mathrm{C}$ for $30 \mathrm{~s}$, followed by 40 cycles of $95^{\circ} \mathrm{C}$ for $5 \mathrm{~s}, 58^{\circ} \mathrm{C}$ for $10 \mathrm{~s}$, and $72^{\circ} \mathrm{C}$ for $10 \mathrm{~s}$, and a final extension at $72^{\circ} \mathrm{C}$ for $5 \mathrm{~min}$. The primers used for real-time PCR analyses are described in Table A3 in Appendix. The expression level of the OsActin gene detected with actin-specific primers was used to standardize the RNA sample for each real-time PCR. The real-time PCR was performed according to the manufacturer's protocol (SYBR Premix Ex Taq ${ }^{\mathrm{TM}}$; Takara) in an ABI PRISM 7500 real-time PCR system.

\section{IN VITRO SYNTHESIS OF dSRNA}

In vitro synthesis of dsRNA was carried out according to the method of Zhai et al. (2009) with minor modifications. DNA templates were synthesized by PCR from rice cDNA and engineered to contain the minimal T7 RNA polymerase promoter sequence

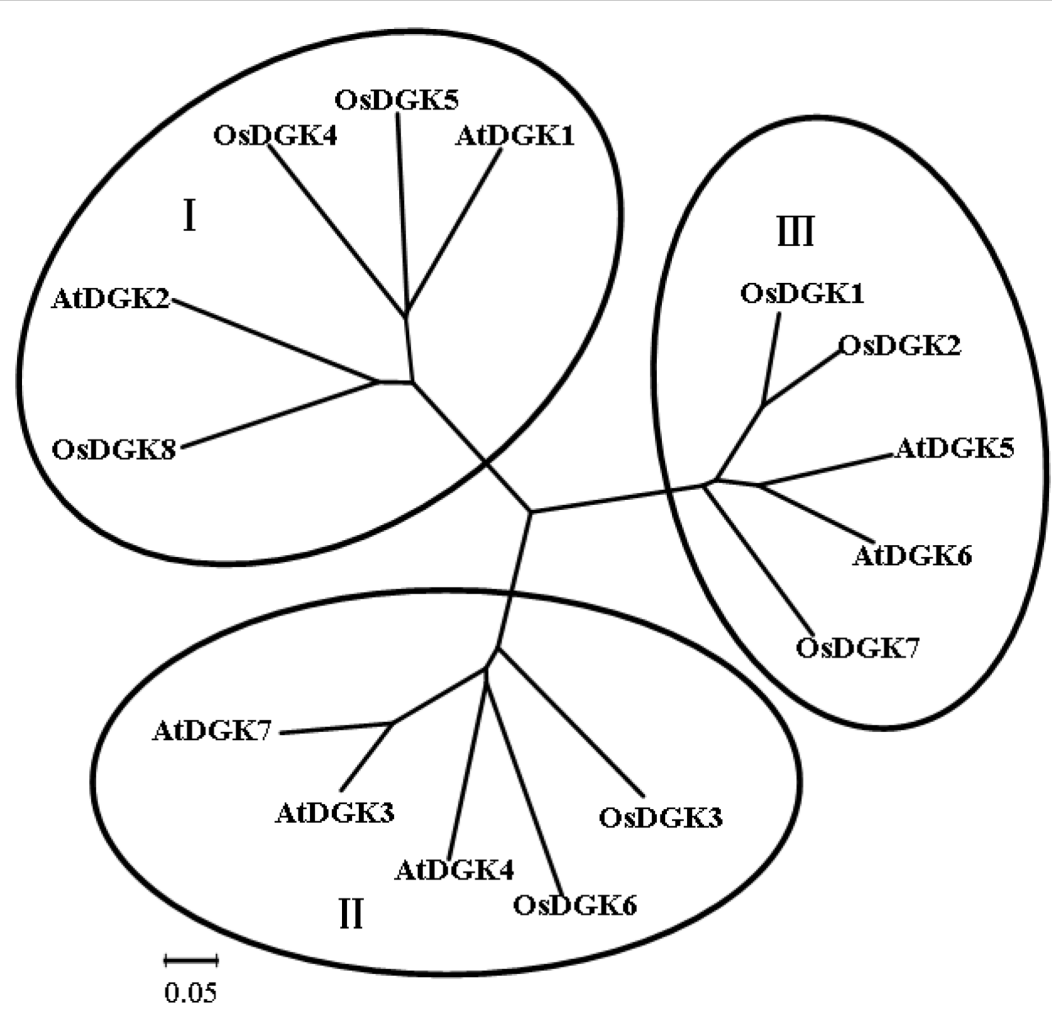

FIGURE 1 | Phylogenetic analysis of the DGK family in rice and Arabidopsis. Accession numbers for the DGKs are as follows: AtDGK1 (At5g07920), AtDGK2 (At5g63770), AtDGK3 (At2g18730), AtDGK4 (At5g57690), AtDGK5 (At2g20900), AtDGK6 (At4g28130), and AtDGK7 (At4g30340); OsDGK1 (Os04g54200), OsDGK2 (Os08g08110), OsDGK3
(Os02g54650), OsDGK4 (Os12g38780), OsDGK5 (Os03g31180), OsDGK6 (Os08g15090), OsDGK7 (Os01g57420), and OsDGK8 (Os12g12260). At, Arabidopsis thaliana; Os, Oryza sativa. The phylogenetic analysis was performed with the neighbor-joining method using the MEGA program. 
(TAATACGACTCACTATAGGGAGG) at both the $5^{\prime}$ and $3^{\prime}$ ends. The primers used to amplify DNA from the targeted genes are listed in Table A4 in Appendix. The PCR conditions were as follows: denaturing at $94^{\circ} \mathrm{C}$ for $5 \mathrm{~min}$, followed by 32 cycles of $94^{\circ} \mathrm{C}$ for $30 \mathrm{~s}, 62^{\circ} \mathrm{C}$ for $45 \mathrm{~s}$, and $72^{\circ} \mathrm{C}$ for $1 \mathrm{~min}$, and a final extension at $72^{\circ} \mathrm{C}$ for $10 \mathrm{~min}$. dsRNAs were synthesized in vitro using the Ribo$\mathrm{MAX}^{\mathrm{TM}}$ Large Scale RNA Production Systems T7 Kit (Promega) according to the manufacturer's recommendations. DNA templates were removed using RNase-free DNase (Promega). dsRNA was purified using the RNeasy kit (Qiagen). The dsRNA was dissolved in DEPC-treated $\mathrm{H}_{2} \mathrm{O}$, and its yield was measured using a UV spectrometer. Typical yields of RNA from $1 \mu \mathrm{g}$ of DNA template were in the 80 - to $100-\mu \mathrm{g}$ range. The dsRNA was separated on a $1 \%$ agarose gel to check its integrity and size.

To prepare the fluorescent dsRNAs, a 40-bp of dsRNA directed against OsPLD $\alpha 1$ was synthesized, and labeled with FAM fluorescence at the $5^{\prime}$ end of sense strand. The sense and antisense strands of dsRNAs were: 5'-AGGCGCCACCAAGGUGUAUUCUACCA UUGAUCUGGAGAAA (sense); 5'-UUUCUCCAGAUCAAUGGU AGAAUACACCUU GGUGGCGCCU (antisense). The transfection of the dsRNA was done according to the protocol by the manufacturer (GenePharma, China). The fluorescence was visualized under a confocal microscope (TCS SP2, Leica, Germany).

\section{TRANSFECTION OF PROTOPLASTS WITH dsRNAs}

Protoplasts $\left(1 \times 10^{6} \mathrm{ml}^{-1}\right)$ in W5 solution were incubated on ice for $30 \mathrm{~min}$. The protoplasts were pelleted and resuspended at $1 \times 10^{6} \mathrm{ml}^{-1}$ in $\mathrm{MMg}$ solution $\left(0.6 \mathrm{M}\right.$ mannitol, $15 \mathrm{mM} \mathrm{MgCl}_{2}$, $4 \mathrm{mM}$ MES, pH 5.6). dsRNA (5-10 $\mu \mathrm{g}$ ) was added to $100 \mu \mathrm{l}$ of protoplasts $\left(1 \times 10^{5}\right.$ in $\mathrm{MMg}$ solution) to which an equal volume of PEG solution [40\% (v/v) PEG4000, 0.4 M mannitol, 0.1 $\mathrm{M} \mathrm{CaCl}_{2}$ ] was gradually added, and the mixture was incubated at room temperature in darkness for $15 \mathrm{~min}$. The transfected protoplasts were collected by centrifugation for $2 \mathrm{~min}$ at $150 \times g$ after diluting the transfection mixture with $600 \mu \mathrm{l}$ of W5 solution. The protoplasts were resuspended in $1 \mathrm{ml}$ of W5 solution and incubated in a six-well culture plate at $28^{\circ} \mathrm{C}$ in darkness for the indicated times.

\section{RT-PCR ANALYSIS OF GENE EXPRESSION IN RNAi PROTOPLASTS}

At the end of the transfection, the protoplasts were collected by centrifugation for $2 \mathrm{~min}$ at $150 \times \mathrm{g}$. Total RNAs were isolated from protoplasts with the Trizol reagent according to the manufacturer's protocol (Takara). Reverse transcription was performed using the Prime Script ${ }^{\mathrm{TM}} \mathrm{RT}$ reagent Kit (Takara). The primers used for RT-PCR analyses are described in Table A2 in Appendix. The genesilencing effect of the dsRNA was visualized in a $1 \%$ agarose gel, comparing the relative expression of RNAi-targeted genes to that of OsGAPDH. All photographs were taken in the Bio-Rad UV-Gel documentation system using Quantity-One analysis software.

\section{GENOMIC SEARCH AND SEOUENCE ANALYSIS}

To identify $D G K$ gene homologs in rice, BLAST searches were performed using the reported sequences of OsDGK (Os04g54200; Zhang et al., 2008) and AtDGK (Gómez-Merino et al., 2004) at the TIGR ${ }^{1}$ and $\mathrm{NCBI}^{2}$ web sites. Sequences of rice and Arabidopsis

${ }^{1}$ http://rice.plantbiology.msu.edu

${ }^{2} \mathrm{http}: / /$ www.ncbi.nlm.nih.gov/
DGK (Gómez-Merino et al., 2004) proteins were aligned using CLUSTAL X (ver. 1.83), and a phylogenetic tree was constructed with the neighbor-joining method using the MEGA program ${ }^{3}$.

\section{DOMAIN ANALYSIS}

Searches for conserved domains in the OsDGK proteins were carried out using SMART ${ }^{4}$ and PFAM $^{5}$

\section{RESULTS}

\section{THE DGK FAMILY IN RICE}

To identify members of the DGK family in rice (O. sativa), sequence information from Arabidopsis and rice was used to perform searches of relevant DNA databases and protein domains. Text searches using the keyword "diacylglycerol kinases" were also performed. Eight putative genes were identified in rice using these approaches. The obtained sequences were further analyzed for their potential to encode a DGK using the programs PFAM and SMART. To study the evolutionary relationships between different DGK members, the MEGA program was used to analyze the phylogenetic relationships of DGKs from rice and Arabidopsis (Figure 1). The results indicate that OsDGKs fall into three phylogenetic clusters, as also described for the AtDGKs (Gómez-Merino et al., 2004). Cluster I comprises $\operatorname{OsDGK}(4,5,8)$, and its closest homologs are $\operatorname{AtDGK}(1,2)$; cluster II contains $\operatorname{OsDGK}(3,6)$, and $\operatorname{AtDGK}(1,4,7)$ are within the same group; finally, the isoforms $\operatorname{OsDGK}(1,2,7)$ fall into cluster III, along with $\operatorname{AtDGK}(5,6)$.

\section{PROTEIN DOMAINS IN OSDGKs}

Domain analyses using the SMART and PFAM databases revealed that the rice DGK family contains a catalytic domain, an accessory domain, a C1 domain, and a PPR domain (Figure 2). In eukaryotic DGKs, the kinase domain contains a conserved catalytic domain with a presumed ATP binding site, and an accessory domain to make contact with the catalytic domain (Gómez-Merino et al.,

\footnotetext{
${ }^{3}$ http://www.megasoftware.net

${ }^{4} \mathrm{http}: / /$ smart.embl-heidelberg.de/

${ }^{5}$ http://pfam.sanger.ac.uk/search.
}

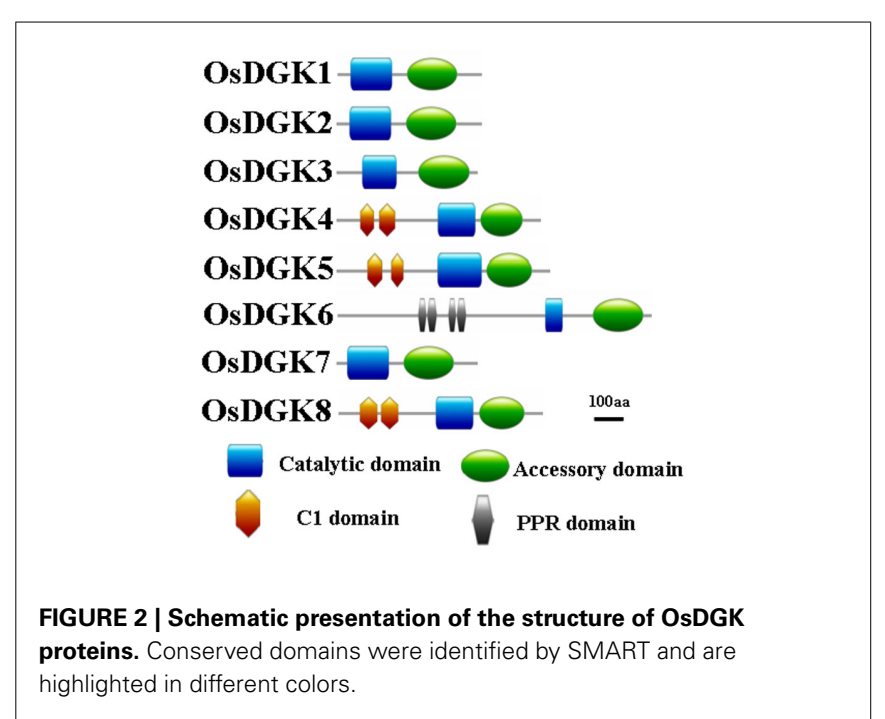


2004; Arisz et al., 2009). The C1 domain contains a tandem Cysrich sequence, which was first identified in $\mathrm{PKC}$ as binding to DAG and phorbol esters (PE; Azzi et al., 1992). This domain was suggested to exist in Arabidopsis DGKs (AtDGK1 and AtDGK2; Gómez-Merino et al., 2004; Arisz et al., 2009). The PPR (pentatricopeptide repeat) domain has been proposed to mediate
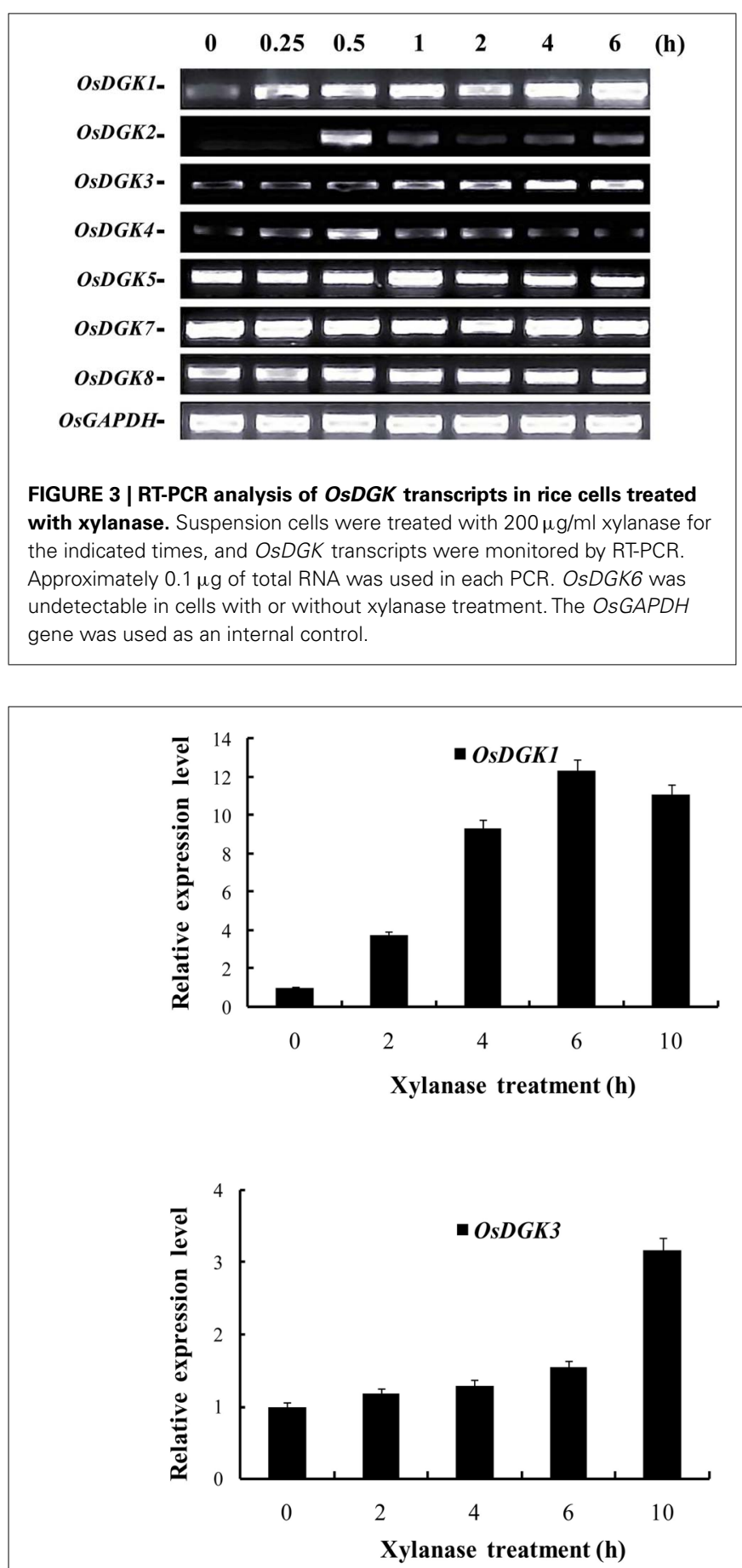

macromolecular interactions. More than 400 genes encoding PPR proteins have been reported to exist in Arabidopsis, and most are predicted to reside in either mitochondria or chloroplasts (Small and Peeters, 2000; Lurin et al., 2004).

All of the OsDGKs were found to contain a catalytic domain and an accessory domain. In addition, $\operatorname{OsDGK}(4,5,8)$ harbor two $\mathrm{C} 1$ domains, whereas OsDGK6 has four putative PPR domains (Figure 2). To our knowledge, among the reported plant DGKs, only OsDGK6 contains a PPR domain. It should be noted that OsDGK6 could be a unique (or putative) OsDGK, according to protein structure analysis (Figure 2), but it was nevertheless grouped into the same cluster (II) as OsDGK3 in a bioinformatics analysis (Figure 1). A similarity analysis revealed the highest similarity between $O s D G K 6$ and $O s D G K 3$ (Table A5 in Appendix). In addition, we isolated all OsDGK cDNAs except for that of OsDGK6. RT-PCR analysis did not detect the OsDGK6 transcript under normal or stress conditions (Figure 3); therefore, the existence and function in rice of this OsDGK have yet to be determined.

\section{XYLANASE-INDUCED EXPRESSION OF OSDGK IN CELLS}

The expression of OsDGK genes in suspension cells was analyzed using RT-PCR. Under control conditions, the transcription of six of the eight OsDGKs (all except for OsDGK2 and OsDGK6) was confirmed. The activation of PLC and DGK pathway has been reported in tomato cells treated with the fungal elicitor xylanase (Laxalt et al., 2007). We therefore explored whether
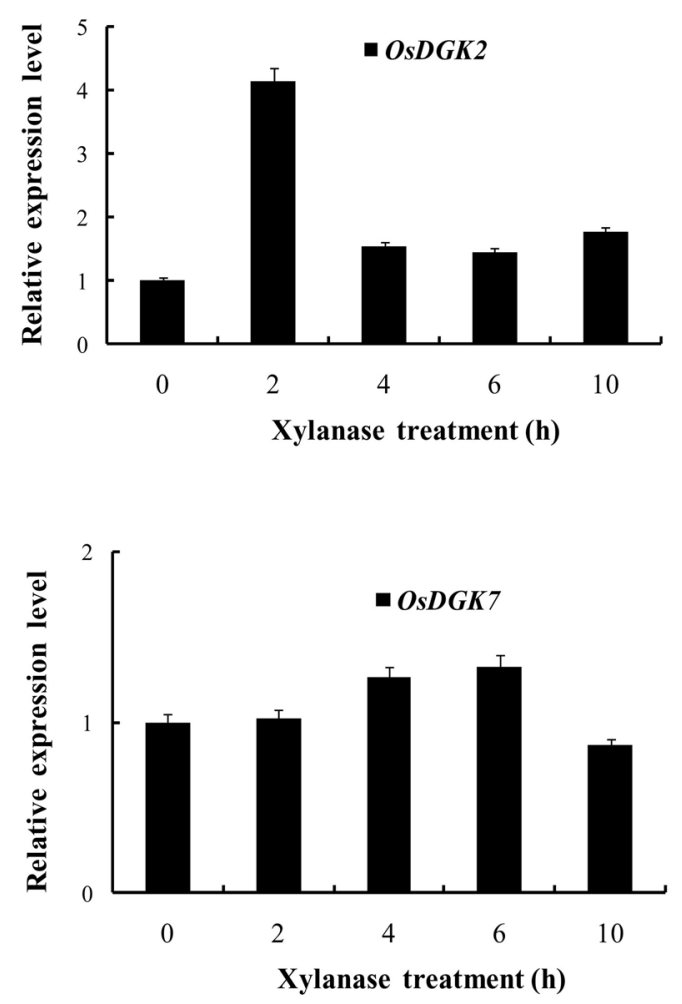

FIGURE 4 | Real-time PCR analysis of the expression of $\operatorname{OsDGK}(\mathbf{1}, \mathbf{2}, \mathbf{3}, \mathbf{7})$. Rice protoplasts were treated with $150 \mu \mathrm{g} / \mathrm{ml}$ xylanase. The transcripts of four OSDGK genes were monitored with real-time PCR. The data shown are averages of three replicates, each with three PCR samples from the same cDNA archive. 
expression of $O s D G K(s)$ are activated by the xylanase. Treatment of cells with $200 \mu \mathrm{g} / \mathrm{ml}$ xylanase led to increases in the transcription of $\operatorname{OsDGK}(1,2,3,4)$. Although the basal transcript levels of $\operatorname{OsDGK}(5,7,8)$ were high, their transcription was not affected by xylanase treatment. The transcription of OsDGK6 was undetectable with or without xylanase (Figure 3).

We selected $\operatorname{OsDGK}(1,2,3)$, which were induced by xylanase, and $O s D G K 7$, which was not induced by xylanase, for further testing with real-time PCR. As shown in Figure 4, the transcription of both $O s D G K 1$ and $O s D G K 3$ gradually increased in xylanasetreated protoplasts. The transcription of OsDGK2 exhibited a sharp peak at $2 \mathrm{~h}$ after the addition of $150 \mu \mathrm{g} / \mathrm{ml}$ xylanase. A peak in the OsDGK2 transcript was also found in the RT-PCR analysis, but it appeared earlier, probably due to the higher concentration of xylanase $(150 \mu \mathrm{g} / \mathrm{ml}$ there $)$ used. The transcription of OsDGK7 was not affected by xylanase under the tested conditions.

\section{TRANSIENT SUPPRESSION OF OsDGK EXPRESSION BY INTRODUCTION OF THE CORRESPONDING dsRNA}

To study the functions of OsDGKs in rice cells, we established methods for dsRNA-induced RNA silencing according to published procedures (Endo et al., 2008; Zhai et al., 2009). Transfection with in vitro-synthesized dsRNAs against target genes into cells resulted in the depletion of their transcripts due to the combined actions of the DICER enzyme and the RNA-induced silencing complex (RISC) enzyme (Figure 5A).

We first selected $O S P L D \alpha 1$ to test because the expression of its mRNA was characterized in our previous work (Shen et al.,

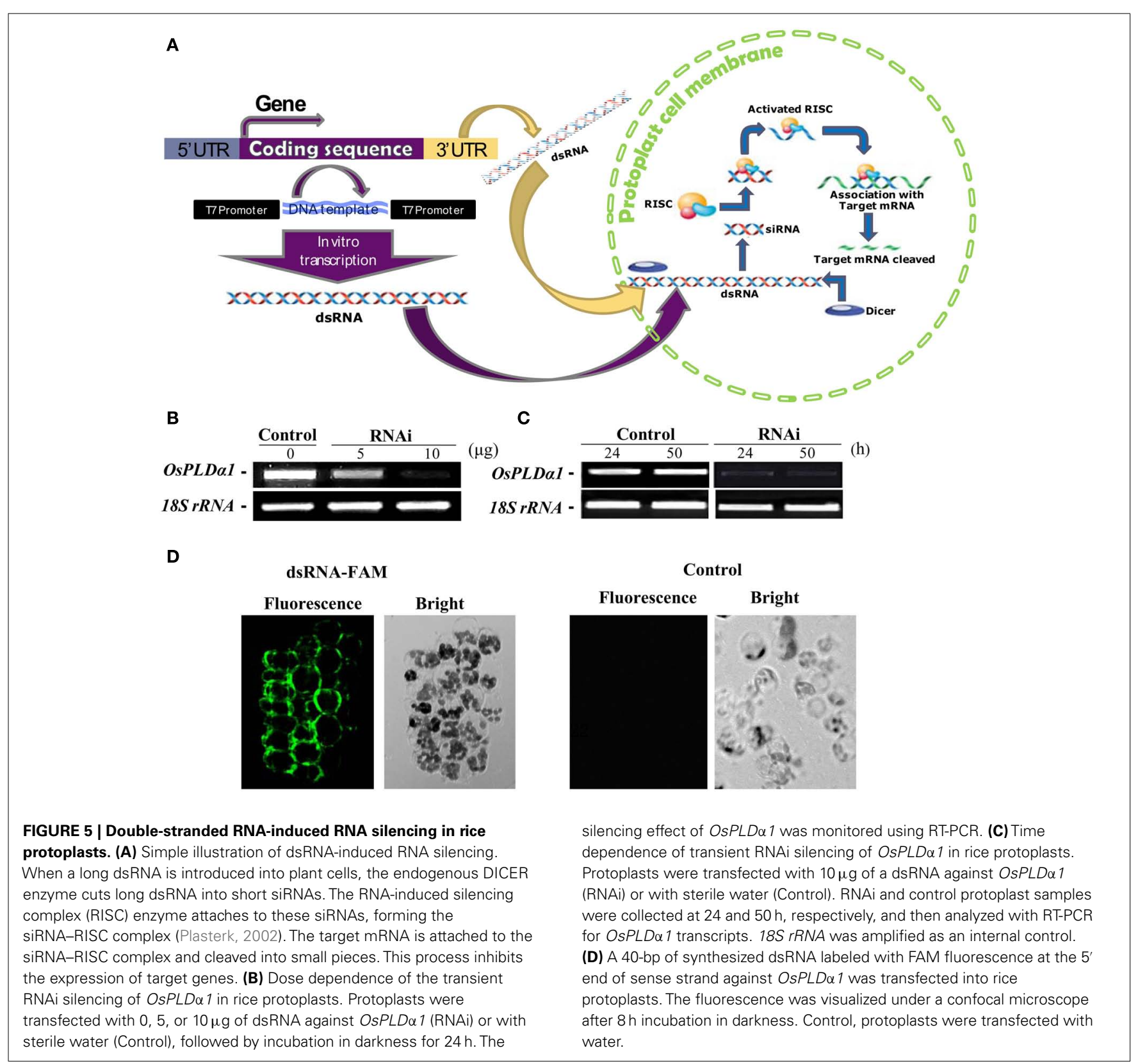


2011). A dsRNA against a 486-bp sequence corresponding to the $O s P L D \alpha 1$ coding sequence was synthesized in vitro. Rice protoplasts were transfected with 5 or $10 \mu \mathrm{g}$ of dsRNA (RNAi) or with sterile water (control, as a mock transfection) and incubated for $24 \mathrm{~h}$ in darkness. The silencing effects of dsRNA were dosedependent (Figure 5B). This inhibition of transcription lasted for at least $50 \mathrm{~h}$ (Figure 5C).

To prove the in vitro-synthesized dsRNA was transported into protoplasts, we synthesized a 40-bp dsRNA labeled with FAM fluorescence at the $5^{\prime}$ end of sense strand against OsPLD $\alpha 1$, and transfected into rice protoplasts. The transfected protoplasts were visualized under a fluorescent microscope after $8 \mathrm{~h}$ incubation in darkness. The fluorescence was found in protoplasts. As a control, no fluorescence was found in the protoplasts transfected with sterile water (Figure 5D). This together with the RT-PCR results suggest that in vitro-synthesized dsRNA had been transported into protoplasts and suppressed gene transcripts.

We then tried to silence expression of the OsDGK family. Two in vitro-synthesized dsRNAs against conserved regions of $\operatorname{OsDGK}(1,2,3,7)$ and $\operatorname{OsDGK}(4,5,8)$ were simultaneously transfected into protoplasts. Mock-transfected protoplasts (control) and two dsRNA-transfected protoplasts (RNAi) were collected for RT-PCR analysis after incubation in darkness for $24 \mathrm{~h}$. The abundance of the OsDGK transcripts after RT-PCR analysis was normalized to the internal standard gene OsGAPDH. As shown in Figure 6A, the transcription of seven OsDGKs was successfully repressed simultaneously by two dsRNAs as compared the mock-transfected control.

We next carried out gene-specific interference of the expression of OsDGKs. RT-PCR analysis was undertaken for OsDGK2, OsDGK3, and OsDGK7 after transient RNAi using in vitrosynthesized dsRNAs corresponding to their $3^{\prime}$-untranslated regions ( $3^{\prime}$-UTRs). The expression of OsDGK2 was repressed significantly by introducing in vitro-synthesized dsRNA directed against its $3^{\prime}$-UTR, whereas the expression of OsDGK3 and OsDGK7 was not affected (Figure 6B). Similarly, gene-specific interference was found for OsDGK3 versus OsDGK2 and OsDGK7, and $O s D G K 7$ versus $O S D G K 2$ and OsDGK3. Taken together, these results suggest that the introduction of dsRNAs is effective for targeted gene silencing in rice protoplasts.

\section{EFFECT OF THE TRANSIENT SILENCING OF OsDGK ON THE TRANSCRIPTION OF GENES RELATED TO STRESS TOLERANCE}

Using the transient RNA interference assay, we next explored the functions of the OsDGKs in stress responses. The WRKY transcriptional factors have been reported to be involved in various stress responses; in particular, overexpression of OsWRKY71 enhances resistance to virulent bacterial pathogens (Liu et al., 2007). Treatment of protoplasts with xylanase $(150 \mu \mathrm{g} / \mathrm{ml})$ induced an increase in the expression of OsWRKY71 and the pathogen-related gene OsNPR1 (Liu et al., 2005). Transient silencing of OsDGKs triggered by the introduction of two dsRNAs corresponding to the conserved regions of $\operatorname{OsDGK}(1,2,3,7)$ and $\operatorname{OsDGK}(4,5,8$; Figure 6A) prevented the increases in expression of OsWRKY71 and OsNPR1 induced by xylanase (Figures 7A,B).

We next asked whether OsDGKs regulate abiotic stress responses. The expression of OsWRKY7 was not affected by salt

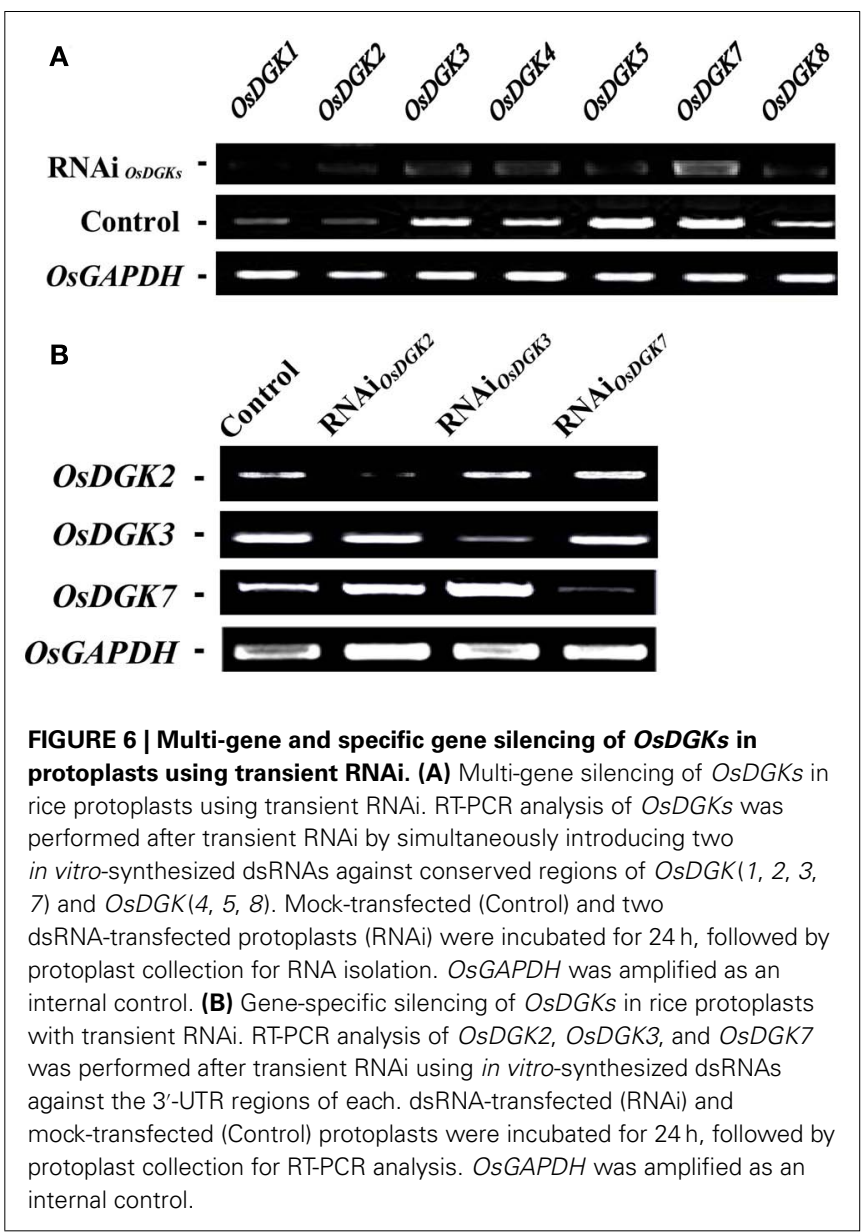

stress; transient silencing of OsDGKs had no effect on OsWRKY7 expression (Figure 8A). However, under salt stress, the expression of OsCIPK15 (CIPK, for calcineurin B-like protein interaction protein kinase) was induced, and the overexpression of OsCIPK15 improved the salt tolerance of rice seedlings (Xiang et al., 2007). An increase in the transcription of OsCIPK 15 was also observed in rice protoplasts exposed to $\mathrm{NaCl}$ solution. This $\mathrm{NaCl}$-induced increase in OsCIPK15 was repressed in cells in which OsDGKs were transiently silenced (Figure 8B). These results suggest that OsDGKs regulate abiotic and biotic stresses through different signaling pathways.

\section{DISCUSSION}

Diacylglycerol kinase phosphorylates DAG to generate PA, serving as a DAG consumer as well as a PA generator. Therefore, DGK is thought to regulate the balance between these two lipid messengers by catalyzing their interconversion (Frere and Paolo, 2009). The mammalian DGKs are a large enzyme family with 10 isozymes, which are subdivided into five groups according to their structural features (Sakane et al., 2007). In the Arabidopsis genome, seven DGK isoforms that form three clusters have been identified (Gómez-Merino et al., 2004). The rice DGKs also group into three clusters (Figure 1). Both Arabidopsis and rice DGKs contain catalytic and accessory domains (Gómez-Merino et al., 2004; Figure 2). $\operatorname{OsDGK}(4,5,8)$ and $\operatorname{AtDGK}(1,2)$ belong 

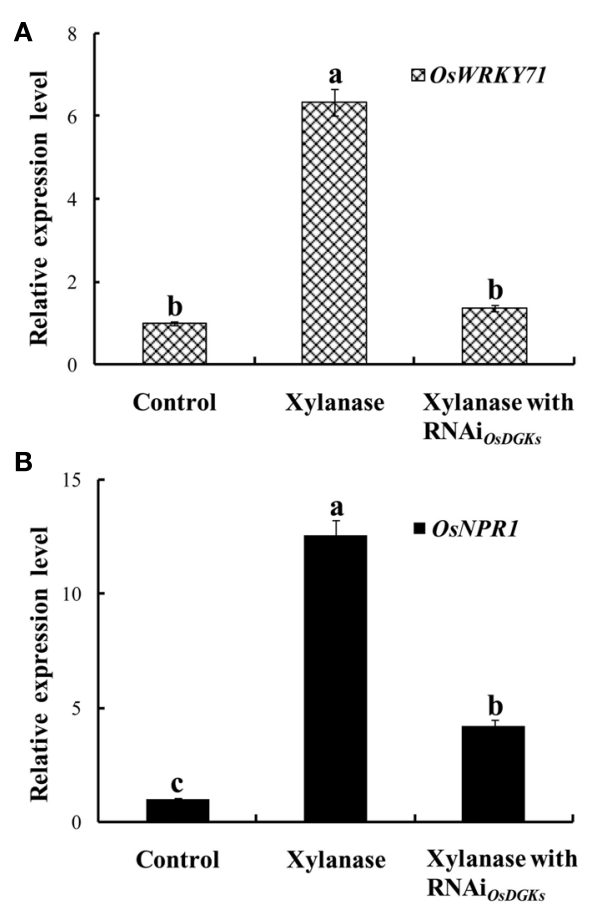

FIGURE 7 | Effects of silencing of OsDGKs on OsWRKY71 and OsNPR1 transcription induced by xylanase in rice protoplasts. After transient RNAi using two in vitro-synthesized dsRNAs against conserved regions of $\operatorname{OsDGK}(1,2,3,7)$ and $\operatorname{OsDGK}(4,5,8)$ for $24 \mathrm{~h}$, the transfected protoplasts were treated with $150 \mu \mathrm{g} / \mathrm{ml}$ xylanase for $6 \mathrm{~h}$. The protoplasts were collected for real-time PCR analysis to detect transcripts of OsWRKY71 (A) and OsNPR1 (B). Values followed by different letters differ significantly $(P<0.01)$.

to cluster I, which possesses two cysteine-rich domains conferring $\mathrm{C} 1$ domains. These domains are absent from other Arabidopsis and rice DGKs (Figure 2; Vaultier et al., 2008). The C1 domain can bind to proteins to modulate the interaction with lipids and proteins (Colon-Gonzalez and Kazanietz, 2006). AtDGK7 still displays kinase activity in the absence of the $\mathrm{C} 1$ domain (GómezMerino et al., 2005), suggesting that this domain is not necessary for its phosphorylation activity (Snedden and Blumwald, 2000). By fusion with fluorescent proteins, the hydrophobic segment in the amino-terminal region upstream of the C1 domain in AtDGK1 and AtDGK2 has been proven to be sufficient and necessary to sequester proteins to ER membranes (Vaultier et al., 2008). However, whether the final localization of full-length DGKs is identical with this segment is unclear. Much less is known about plant DGKs than animal DGKs.

Transient RNA interference caused the decreased expression of unique and multiple $O s D G K$ genes in rice protoplasts (Figure 6). This approach has also been successfully used in Arabidopsis and

\section{REFERENCES}

Arisz, S. A., Testerink, C., and Munnik, T. (2009). Plant PA signaling via diacylglycerol kinase. Biochim. Biophy. Acta 179, 869-875.

Azzi, A., Boscoboinik, D., and Hensey, C. (1992). The protein kinase
C family. Eur. J. Biochem. 208, 547-557.

Chen, J., Zhang, W. D., Song, F. M., and Zheng, Z. (2007). Phospholipase C/diacylglycerol kinasemediated signalling is required for benzothiadiazole-induced oxidative
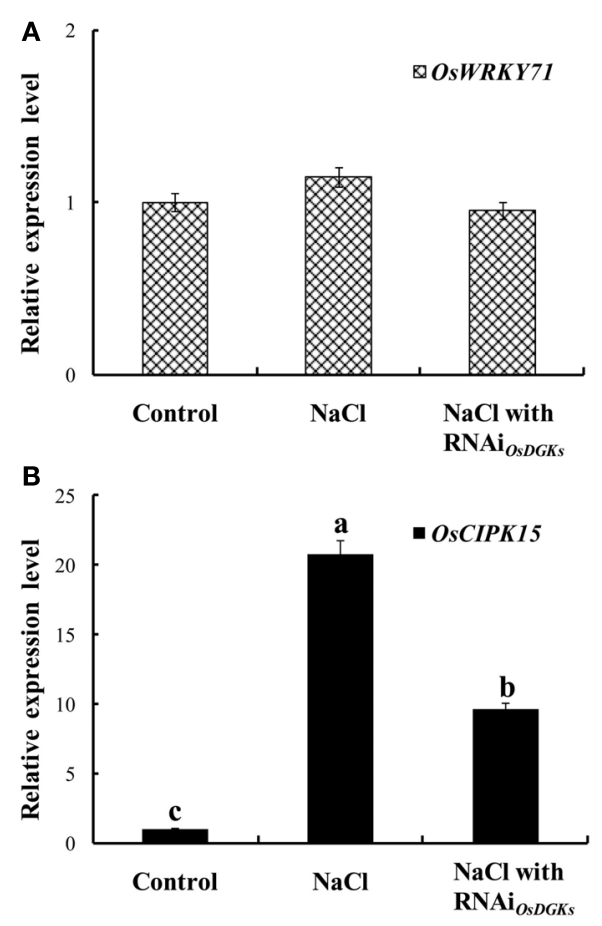

FIGURE 8 | Effects of silencing of OsDGKs on transcription of OsWRKY71 and OsCIPK15 induced by $\mathrm{NaCl}$ in rice cells. Following transient RNAi using two in vitro-synthesized dsRNAs against conserved regions of $\operatorname{OsDGK}(1,2,3,7)$ and $\operatorname{OsDGK}(4,5,8)$ for $24 \mathrm{~h}$, the transfected protoplasts were treated with $50 \mathrm{mM} \mathrm{NaCl}$ for $6 \mathrm{~h}$. The protoplasts were collected for real-time PCR analysis to detect transcripts of OsWRKY71 (A) and OSCIPK15 (B). Values followed by different letters differ significantly $(P<0.01)$.

Zinnia (Endo et al., 2008; Zhai et al., 2009). Introduction of in vitro-synthesized dsRNAs corresponding to the cellulose synthesis gene CesA into Zinnia cells repressed the expression of Zinnia CesA homologs. The repression phenocopies Arabidopsis cellulose synthase mutants that have defects in secondary cell wall synthesis and increased abnormal tracheary elements (Endo et al., 2008). In our work, repression of multiple OsDGK genes impaired stressinduced gene expression (Figures 7 and 8). The combination of transient RNA interference and DGK mutants will help to fully elucidate the functions of DGKs in plants.

\section{ACKNOWLEDGMENTS}

This work was supported by grants from the National Science Foundation of China $(31171461,30625027)$, the Ministry of Science and Technology of China (2012CB11420), the Fundamental Research Funds for the Central Universities (KYT201001), the Education Department of Jiangsu (200910, PAPD), and from the Youth Sci-Tech Innovation Fund, NJAU (KJ09019).

burst and hypersensitive cell death in rice suspension-cultured cells. Protoplasma 230, 13-21.

Colon-Gonzalez, F., and Kazanietz, M. G. (2006). C1 domains exposed: from diacylglycerol binding to protein-protein interactions.
Biochim. Biophys. Acta 1761, 827-837.

Endo, S., Pesquet, E., Tashiro, G., Kuriyama, H., Goffner, D., Fukuda, H., and Demura, T. (2008). Transient transformation and RNA silencing in Zinnia tracheary element 
differentiating cell cultures. Plant J. $53,864-875$.

Frere, S. G., and Paolo, G. D. (2009). A lipid kinase controls the maintenance of dendritic spines. $E M B O J$. 28, 999-1000.

Gómez-Merino, F. C., Arana-Ceballos, F. A., Trejo-Tëllez, L. I., Skirycz, A., Brearley, C. A., Dörmann, P., and Mueller-Roeber, B. (2005). Arabidopsis AtDGK7, the smallest member of plant diacylglycerol kinases (DGKs), displays unique biochemical features and saturates at low substrate concentration. J. Biol. Chem. 280, 34888-34899.

Gómez-Merino, F. C., Brearley, C. A., Ornatowska, M., Abdel-Haliem, M. E., Zanor, M. I., and MuellerRoeber, B. (2004). AtDGK2, a novel diacylglycerol kinase from Arabidopsis thaliana, phosphorylates 1-stearoyl-2-arachidonoyl-snglycerol and 1,2-dioleoyl-sn-glycerol and exhibits cold-inducible gene expression. J. Biol. Chem. 279, 8230-8241.

Hong, Y., Zhang, W., and Wang, X. (2010). Phospholipase D and phosphatidic acid signaling in plant response to drought and salinity. Plant Cell Environ. 33, 627-635.

Kamada, Y., and Muto, S. (1991). Ca ${ }^{2+}$ regulation of phosphatidylinositol turnover in the plasma membrane of tobacco suspension culture cells. Biochim. Biophys. Acta 1093, 72-79.

Katagiri, T., Mizoguchi, T., and Shinozaki, K. (1996). Molecular cloning of a cDNA encoding diacylglycerol kinase (DGK) in Arabidopsis thaliana. Plant Mol. Biol. 30, 647-653.

Laxalt, A. M., Raho, N., Have, A. T., and Lamattina, L. (2007). Nitric oxide is critical for inducing phosphatidic acid accumulation in xylanaseelicited tomato cells. J. Biol. Chem. 282, 21160-21168.

Li, M., Hong, Y., and Wang, X. (2009). Phospholipase D- and phosphatidic acid-mediated signaling in plants. Biochim. Biophys. Acta 1791, 927-935.

Liu, X., Bai, X., Wang, X., and Chu, C. (2007). OsWRKY71, a rice transcription factor, is involved in rice defense response. J. Plant Physiol. 164, 969-979.

Liu, X. Q., Bai, X. Q., Qian, Q., Wang, X. J., Chen, M. S., and Chu, C. C. (2005). OsWRKY03, a rice transcriptional activator that functions in defense signaling pathway upstream of OsNPR1. Cell Res. 15, 593-603.

Lundberg, G. A., and Sommarin, M. (1992). Diacylglycerol kinase in plasma membranes from wheat. Biochim. Biophys. Acta 1123 177-183.

Lurin, C., Andrés, C., Aubourg, S., Bellaoui, M., Bitton, F., Bruyère, C., Caboche, M., Debast, C., Gualberto, J., Hoffmann, B., Lecharny, A., Ret, L. M., Martin-Magniette, M. L., Mireau, H., Peeters, N., Renou, J. P., Szurek, B., Taconnat, L., and Small, I. (2004). Genome-wide analysis of Arabidopsis pentatricopeptide repeat proteins reveals their essential role in organelle biogenesis. Plant Cell 16, 2089-2103.

Munnik, T., and Testerink, C. (2009). Plant phospholipid signaling: "in a nutshell". J. Lipid Res. 50, S260-S265.

Plasterk, R. H. A. (2002). RNA silencing: the genome's immune system. Science 296, 1263-1265.
Sakane, F., Imai, S., Kai, M., Yasuda S., and Kanoh, H. (2007). Diacylglycerol kinases: why so many of them? Biochim. Biophy. Acta 1771, 793-806.

Sheen, J. (2001). Signal transduction in maize and Arabidopsis mesophyll protoplasts. Plant Physiol. 127, 1466-1475.

Shen, P., Wang, R., Jing, W., and Zhang, W. (2011). Rice phospholipase Do is involved in salt tolerance by the mediation of $\mathrm{H}+$-ATPase activity and transcription. J. Int. Plant Biol. 53, 289-299.

Small, I. D., and Peeters, N. (2000) The PPR motif-a TPR-related motif prevalent in plant organellar proteins. Trends Biochem. Sci. 25, 46-47.

Snedden, W. A., and Blumwald, E. (2000). Alternative splicing of a novel diacylglycerol kinase in tomato leads to a calmodulin-binding isoform. Plant J. 24, 317-326.

Vaultier, M. N., Cantrel, C., Guerbette, F., Boutte, Y., Vergnolle, C., Cicek, C., Bolte, S., Zachowski, A., and Ruelland, E. (2008). The hydrophobic segment of Arabidopsis thaliana cluster I diacylglycerol kinases is sufficient to target the proteins to cell membranes. FEBS Lett. 582, 1743-1748.

Wissing, J. B., and Wagner, K. G. (1992), Diacylglycerol kinase from suspension cultured plant cells: characterization and subcellular localization. Plant Physiol. 98, 1148-1153.

Xiang, Y., Huang, Y., and Xiong, L. (2007). Characterization of stressresponsive CIPK genes in rice for stress tolerance improvement. Plant Physiol. 144, 1416-1428.

Zhai, Z., Sooksa-Nguan, T., and Vatamaniuk, O. K. (2009). Establishing RNA interference as a reverse-genetic approach for gene functional analysis in protoplasts. Plant Physiol. 149, 642-652.

Zhang, W., Chen, J., Zhang, H., and Song, F. M. (2008). Overexpression of a rice diacylglycerol kinase gene OsBIDK1 enhances disease resistance in transgenic tobacco. Mol. Cells 26, 258-264.

Zhang, W., Wan, X., Hong, Y., Li, W., and Wang, X. (2010). "Plant phospholipase." in Lipid Signaling in Plants, Plant Cell Monographs, Vol. 16, ed. D. T. Munnik (Heidelberg: SpringerVerlag), 39-62.

Conflict of Interest Statement: The authors declare that the research was conducted in the absence of any commercial or financial relationships that could be construed as a potential conflict of interest.

Received: 24 December 2011; accepted: 13 March 2012; published online: 29 March 2012.

Citation: Ge H, Chen C, Jing W, Zhang $Q$, Wang $H$, Wang $R$ and Zhang $W$ (2012) The rice diacylglycerol kinase family: functional analysis using transient RNA interference. Front. Plant Sci. 3:60. doi: 10.3389/fpls.2012.00060

This article was submitted to Frontiers in Plant Physiology, a specialty of Frontiers in Plant Science.

Copyright $\odot 2012$ Ge, Chen, Jing, Zhang, Wang, Wang and Zhang. This is an open-access article distributed under the terms of the Creative Commons Attribution Non Commercial License, which permits non-commercial use, distribution, and reproduction in other forums, provided the original authors and source are credited. 


\section{APPENDIX}

\section{Table A1 | K3 medium used for rice protoplast system.}

K3 medium

$10 \times$ B5 Macro (1 I)

$100 \times$ B5 Micro(I) (1 I)

$1000 \times$ B5 Micro(II) (1 I)

$100 \times$ B5 Vitamins (1 I)
$10 \times$ B5 Macro, $100 \times$ B5 micro (I), $1000 \times$ B5 micro (II), $100 \times$ B5 vitamins, $200 \times$ MES $(0.1 \mathrm{~g} / \mathrm{ml}), 500 \times \mathrm{myo}-\mathrm{inositol}$ $(0.05 \mathrm{~g} / \mathrm{ml}), 100 \times \mathrm{NH}_{3} \mathrm{NO}_{3}(25 \mathrm{mg} / \mathrm{ml}), 100 \times \mathrm{CaCl}_{2}(75 \mathrm{mg} / \mathrm{ml}), 100 \times$ xylose $(25 \mathrm{mg} / \mathrm{ml}), 0.4 \mathrm{M}$ d-mannitol. pH is adjusted to 5.6 by $1 \mathrm{M} \mathrm{KOH}$.

$\mathrm{KNO}_{3} 25 \mathrm{~g},\left(\mathrm{NH}_{4}\right)_{2} \mathrm{SO}_{4} 1.34 \mathrm{~g}, \mathrm{CaCl}_{2} \cdot 2 \mathrm{H}_{2} \mathrm{O} 1.5 \mathrm{~g}, \mathrm{MgSO}_{4} \cdot 7 \mathrm{H}_{2} \mathrm{O} 2.5 \mathrm{~g}, \mathrm{NaH}_{2} \mathrm{PO}_{4} \cdot \mathrm{H}_{2} \mathrm{O} 1.5 \mathrm{~g}$.

$\mathrm{MnSO}_{4} \cdot \mathrm{H}_{2} \mathrm{O} 0.78 \mathrm{~g}, \mathrm{ZnSO}_{4} \cdot 7 \mathrm{H}_{2} \mathrm{O}, 0.2 \mathrm{~g}, \mathrm{H}_{3} \mathrm{BO}_{3} 0.3 \mathrm{~g}, \mathrm{KI} 0.075 \mathrm{~g}$.

$\mathrm{NaMoO}_{4} \cdot 2 \mathrm{H}_{2} \mathrm{O} 0.250 \mathrm{~g}, \mathrm{CuSO}_{4} \cdot 5 \mathrm{H}_{2} \mathrm{O} 25 \mathrm{mg}, \mathrm{CoCl}_{2} \cdot 6 \mathrm{H}_{2} \mathrm{O} 25 \mathrm{mg}$.

Vitamin $\mathrm{B} 1$ (thiamine-HCl) $1 \mathrm{~g}$, vitamin $\mathrm{B} 6$ (pyridoxine- $\mathrm{HCl}$ ) $0.1 \mathrm{~g}$, nicotinic acid $0.1 \mathrm{~g}$.

K3 medium was prepared according to the method by Chen et al. (2006) with minor modification.

Table A2 | List of oligos used for RT-PCR analysis.

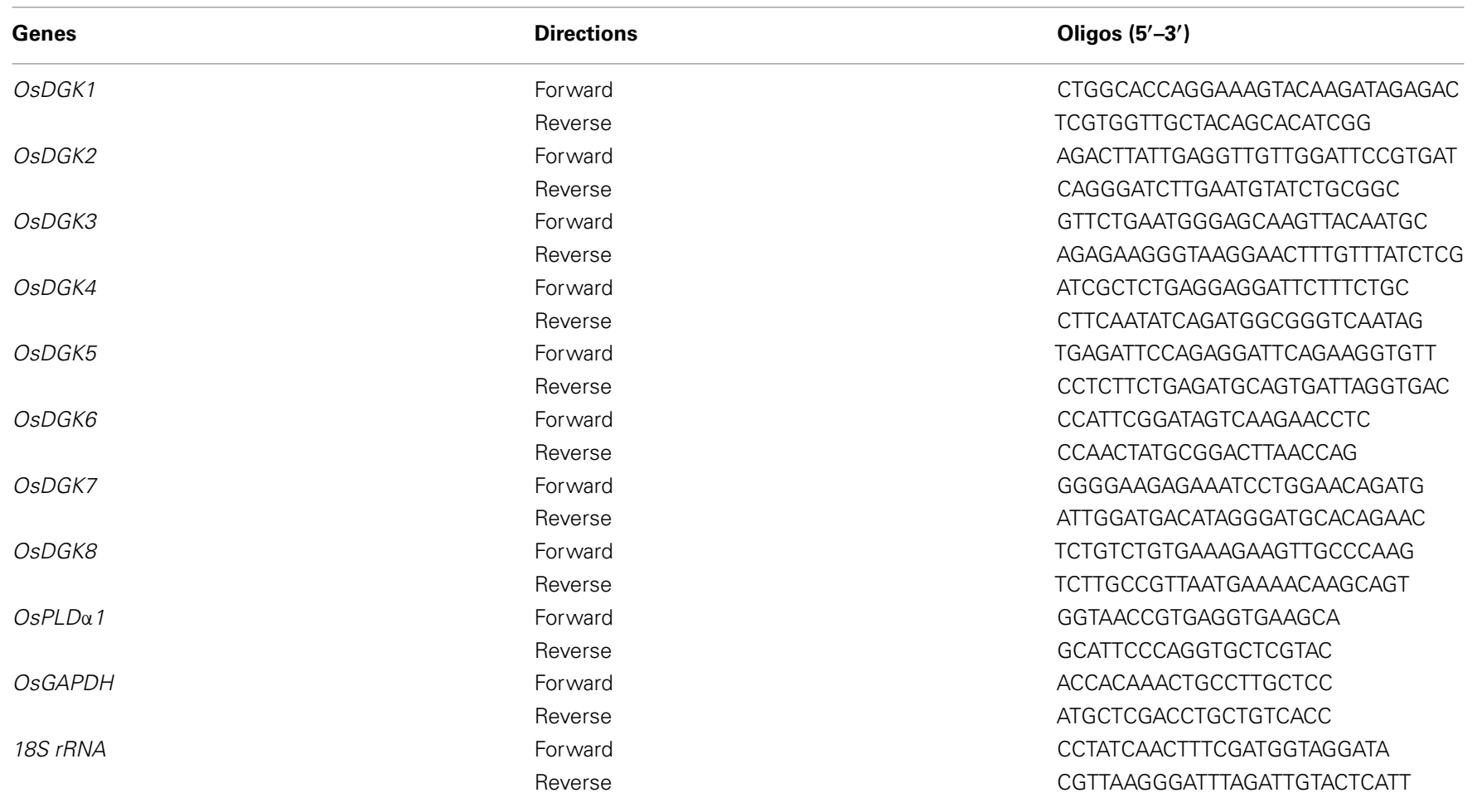


Table A3 | List of oligos used for real-time PCR analysis.

\begin{tabular}{|c|c|c|}
\hline Genes & Directions & Oligo $\left(5^{\prime}-3^{\prime}\right)$ \\
\hline \multirow[t]{2}{*}{ OsDGK1 } & Forward & GGCTGCTTGGTGTAGTTAGTG \\
\hline & Reverse & TCTTGGTCAGTGGTTGGGTT \\
\hline OsDGK2 & Reverse & CACGGAATCCAACAACCTCAAT \\
\hline OsDGK3 & Forward & GAATGGGAGCAAGTTACAATG \\
\hline OsDGK7 & Reverse & CAGCGAATGAGGCAAATCCA \\
\hline \multirow[t]{2}{*}{ OsWRKY71 } & Forward & CGCCGACCCATCCGACCTCA \\
\hline & Reverse & TCTTGACAGGGCAGGCGGGA \\
\hline \multirow[t]{2}{*}{ OsNPR1 } & Forward & CCCGCGATGTTCGAACGTGC \\
\hline & Reverse & CGACGAGAGCCCCGACCTGT \\
\hline
\end{tabular}

Table A4 | List of oligos used in amplifying DNA templates for in vitro dsRNA synthesis.

\begin{tabular}{|c|c|c|c|}
\hline Targeted Gene & Directions & Oligos $\left(5^{\prime}-3^{\prime}\right)$ & Size of dsRNA (bp) \\
\hline \multirow[t]{2}{*}{ OsPLD 1} & Forward & GCTTAATACGACTCACTATAGGGAGGTTGACGATGAGTACATCATCATCGG & 486 \\
\hline & Reverse & GCTTAATACGACTCACTATAGGGAGGCTATGAGGTGAGGATGGGGGGCATG & \\
\hline OsDGKs $(1,2,3,7)$ & Reverse & GCTTAATACGACTCACTATAGGGAGGGGCTGCTTCCATGGCTCCCC & \\
\hline OsDGKs $(4,5,8)$ & Forward & GCTTAATACGACTCACTATAGGGAGGCGCGCGCAGAGGTTAGCTCA & 229 \\
\hline OsDGK2 (3'-UTR) & Reverse & GCTTAATACGACTCACTATAGGGAGGTCTGCTGAACAATAAACAAGAAATC & \\
\hline \multirow[t]{2}{*}{ OsDGK3 (3'-UTR) } & Forward & GCTTAATACGACTCACTATAGGGAGGAATCAGGGTCGTATTCTAGATCGTT & 303 \\
\hline & Reverse & GCTTAATACGACTCACTATAGGGAGGCATAACAGGAGGGGAAATCTGAGTA & \\
\hline \multirow[t]{2}{*}{ OsDGK7 (3'-UTR) } & Forward & GCTTAATACGACTCACTATAGGGAGGTGACGAGGTTTTGTACGTATGGCTG & 342 \\
\hline & Reverse & GCTTAATACGACTCACTATAGGGAGGCGTGGAGGTATATTCTGCGGGTAGT & \\
\hline
\end{tabular}

Table A5 | Amino acid sequence identities among OsDGKs.

\begin{tabular}{|c|c|c|c|c|c|c|c|c|}
\hline & OsDGK1 & OsDGK2 & OsDGK3 & OsDGK4 & OsDGK5 & OsDGK6 & OsDGK7 & OsDGK8 \\
\hline OsDGK1 & & 81 & 32 & 27 & 25 & 29 & 63 & 25 \\
\hline OsDGK2 & & & 31 & 25 & 25 & 29 & 62 & 24 \\
\hline OsDGK4 & & & & & 59 & 17 & 25 & 50 \\
\hline OsDGK5 & & & & & & 16 & 23 & 52 \\
\hline OsDGK8 & & & & & & & & \\
\hline
\end{tabular}

\section{REFERENCE}

Chen, S., Tao, L., Zeng, L., Vega-Sanchez, M. E., Umemura, K., Wang, G. L. (2006). A highly efficient transient protoplast system for analyzing defence gene expression and protein-protein interactions in rice. Mol. Plant Pathol. 7, 417-427. 